\title{
Spondyloarthropathies: are there any differences between Caucasians and south- east Asians living in north-east London in terms of disease onset and progression subject to cultural, social and treatment applications
}

Objective: To compare PSpo patients with those with predominantly peripheral disease. Method: Retrospective cross-sectional analysis of 155 patients with PsA.

Results: 127 patients (81.9\%) had peripheral and 28 patients (18\%) had axial disease. PSpo patients had greater HLAB27 and Uveitis percentages (32\%, 14\% successively) than those with peripheral disease. $64 \%$ of patients subsequently developed peripheral joint involvement predominantly oligo-arthritis pattern (72\%).

Conclusion: further prospective observational studies comparing peripheral and axial PsA are required to elucidate our observation.

Keywords: psoriatic arthritis • spondylitis • peripheral arthritis

\section{Introduction}

It is estimated that inflammatory arthritis affects $2-3 \%$ of the general population and $6-42 \%$ among patients with a diagnosis of psoriasis [1]. Spondylitis is common in patients with PsA and the literature suggests that it can be combined with peripheral involvement [2]. This has not been extensively investigated and importantly, there is a strict classification that segments either spinal or peripheral involvement as exclusive entities.

In 1973 Moll and Wright classified spinal involvement as part of a PsA spectrum [3]. The phenotype resembled Ankylosing Spondylitis, but this subset has been little investigated. In both clinical practice and trials investigators refer to both spinal and predominantly peripheral involvement as being 'Ankylosing Spondylitis' rather than a variant of Spondylitic Psoriatic Arthritis.

\section{Aim}

In this study we sought to
- Study a particular cohort of patients with $\mathrm{SpA}$ and compare their disease and biochemical characteristics with that of peripheral predominant PsA patients

- Assess how this group evolved over time by further review of their medical notes

\section{Statistical analysis}

Data was analyzed using the computer program SPSS (Statistical Package for Social Sciences) version release 16.0 to obtain descriptive statistics including frequency analysis. One way ANOVA, Chi-square, Kruskal-Wallis or Mann-Whitney test was performed for statistical comparison. Correlation was evaluated using Spearman correlation test, algorithmic transformation and linear regression analysis. $\mathrm{P}$ value is considered significant at $\leq 0.05$.

\section{Methods}

A retrospective cross sectional analysis of medical records of 155 patients with psoriatic arthritis, defined in accordance with the CAPSAR criteria [4].
Aicha Bouraoui*, Euthalia Roussou, Sree Sakthibalan \& Khalifa Boukadida

Southend University Hospital NHS Foundation Trust, UK

*Author for correspondence: aicha.bouraoui@nhs.net 
The records analysed were part of a larger study aiming to assess Spondylo-arthropathies among different ethnic groups in the region of North East London. A semi-validated questionnaire called LoRoS (London registry of spondyloarthropathy) was used to collect data in an identical manner descried before in the literature [5].

Inclusion criteria for patients were for anyone above the age of 18 able to consent who presented with evidence of Inflammatory back pain and/or Psoriasis, uveitis, enthesitis, Achilles tendinosis, inflammatory bowel disease and arthritis. Exclusion criteria were inability to consent and poor understanding of the English language.

The data collection of the initial questionnaire took place around the time of presentation to our secondary care Rheumatology department with variable timeline related to disease onset.

Statistical analysis took place using t-test in which a 95\% confidence interval (CI) level and $<0.05$ statistical significance was used to assess difference between axial and peripheral disease groups. Written informed Consents were obtained from all patients whose data were collected using LoRoS questionnaire and the study gained ethics approval (REC:07/ H0701/74) [5].

\section{Results}

From a total of 424 patients with spondylitis who provided baseline data in the LoRoS questionnaire at the time of this analysis, 155 patients were identified to have PsA. A further clinical review of those 155 patients took place using the ASAS criteria aiming to assess the clinical characteristics of the disease.

The mean age of the total group of 155 patients was 56 years (y) (range 22-84 y). Most patients $107(69 \%)$ were female. Looking at clinical presentation from the total of 155 patients defined as having PsA, 127 patients (81.9\%) had peripheral disease and 28 patients (18\%) had axial disease defined as psoriatic spondylitis $(\mathrm{p}<0.05)$. Table 1 shows the analysis of disease characteristics (clinical, social, biochemical) between the 2 groups and the statistical significance levels between the 2 groups.

The records of the 28 patients with psoriatic spondylitis were scrutinized further and interestingly among the 28 patients with axial disease at presentation 18 of 28 (64.2\%) developed peripheral joint involvement predominantly in the of oligo-arthritis pattern as shown in Figure 1, while smaller percentage developed symmetrical polyarthritis $(22 \%)$ and distal inter-phalangeal joint (DIP) involvement.

\section{Table 1. Comparison of peripheral and axial disease in patients with confirmed PsA}

\begin{tabular}{|c|c|c|c|}
\hline Data & Peripheral PsA & $\begin{array}{c}\text { Psoriatic } \\
\text { spondylitis }\end{array}$ & Statistical significance \\
\hline Disease type $((\mathrm{N})=$ number of patients $)$ & $\mathrm{N}=127$ & $\mathrm{~N}=28$ & $P(<0.05)$ \\
\hline Age (years) & $56(+/-14)$ & $57.6(10.5)$ & $0.5(\mathrm{NS})$ \\
\hline Age symptom (years) & $38.29(+/-13.82)$ & $37.1(11.5)$ & $0.6(\mathrm{NS})$ \\
\hline Age diagnosis (years) & $43.3(13.66)$ & $45.4(9.8)$ & $0.4(\mathrm{NS})$ \\
\hline Delay of diagnosis(years) & $3.62(6.59)$ & $6.6(7.2)$ & $0.03(\mathrm{SS})$ \\
\hline Female/ male & $89 / 38$ & $18 / 10$ & $0.5(\mathrm{NS})$ \\
\hline Caucasian \%(n) & $64 \%(82)$ & $46.4 \%(13)$ & $0.07(\mathrm{NS})$ (trend) \\
\hline Asian \%(n) & $23 \%(38)$ & $35.7 \%(10)$ & 0.5 (NS) \\
\hline *Others \%(n) & $13 \%(6)$ & $14.2 \%(4)$ & $0.06(\mathrm{NS})$ (trend) \\
\hline ESR (+ sd) & $18(15.4)$ & $18.2(14.15)$ & 0.9 (NS) \\
\hline CRP (+sd) & $6.5(4.5)$ & $8.6(9.57)$ & $0.07(\mathrm{NS})$ (trend) \\
\hline HLA B27\%(n) & $1.57 \%(2)$ & $32.1 \%(9)$ & $0.00(\mathrm{SS})$ \\
\hline Uveitis \%(n) & $8 \%(4)$ & $14.2 \%(4)$ & $0.01(\mathrm{SS})$ \\
\hline IBP\%(n) & $17.3 \%(22)$ & $42.8 \%(12)$ & $0.003(\mathrm{SS})$ \\
\hline Arthritis \%(n) & $81 \%(103)$ & $64.2 \%(18)$ & 0.05 (NS) \\
\hline Enthesitis \%(n) & $44 \%(56)$ & $53.5 \%(15)$ & $0.3(\mathrm{NS})$ \\
\hline Psoriasis(at stage of diagnosis) \%(n) & $55.11 \%(70)$ & $50 \%(14)$ & $0.6(\mathrm{NS})$ \\
\hline BASFI (+ sd) & $5.21(2.48)$ & $5.7(2.3)$ & $0.3(\mathrm{NS})$ \\
\hline BASDAI (+ sd) & $6.25(1.97)$ & $7.04(1.7)$ & 0.05 (NS) \\
\hline Smoking \%(n) & $29(37)$ & $35.7 \%(10)$ & $0.4(\mathrm{NS})$ \\
\hline Alcohol \%(n) & $53.5 \%(68)$ & $53.5 \%(15)$ & $1(\mathrm{NS})$ \\
\hline Family history \%(n) & $21.2 \%(r 27)$ & $14.2 \%(4)$ & $0.4(\mathrm{NS})$ \\
\hline
\end{tabular}




\section{$\%$ of peripheral PsA Subtypes in patients with psoriatic spondylitis}

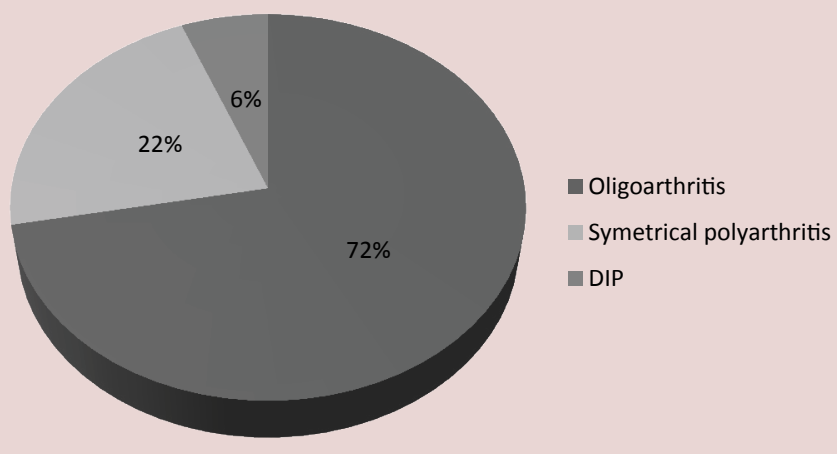

Figure 1. Showing the percentage of PsA subtypes that developed in those patients with initial presentation as Psoriatic Spondylitis.

\section{Discussion}

This is a retrospective analysis of patients with PsA who presented with IBP and manifestations of spinal involvement to secondary care. Our results showed that Peripheral PsA is more prevalent than psoriatic spondylitis with a 1 to 4.5 ratio of those with PsA and IBP at presentation and no peripheral disease.

Both groups were found to have similar epidemiological characteristics. HLA B27 phenotype and Uveitis were associated with patients in the psoriatic spondylitis group who had higher BASDAI scores compared to peripheral PsA. This has been demonstrated in previous studies, which suggest that HLA-B27 is not an only marker for the susceptibility of psoriatic spondylitis but also of an earlier and severe onset of psoriasis and arthritis [6].

Interestingly, upon further analysis we identified that a proportion of those patients subsequently progressed by developing peripheral disease predominantly of the oligo articular pattern. To the best of our knowledge this has not been reported before therefore we are unable to compare our data with data from other centres.

Our study raises the dilemma related to the overlap in the exclusive classification criterion for axial and peripheral SpA (ASAS). This also unveils the differences in the use of CASPAR or ASAS for the diagnosis of Spondyitis associated with PsA $[4,7]$.

Despite the significant development in classification criteria of PsA, it remains difficult to clearly distinguish between the two groups in daily clinical practice. Further research into the nature of a possible spectrum of disease needs to be considered.

When it comes to the terminology used, although we went for term psoriatic spondylitis, there are no worldwide agreed definitions. In this study, the term Psoriatic Spondylitis was used to group the patients with axial involvement. We acknowledge that there are no clear worldwide definitions for such involvement. Patients with predominantly axial symptoms should strictly be diagnosed with axial spondylitis- with or without psoriasis. However, other authors have labeled psoriatic patients with axial symptoms under the term axial PsA [8].

Our data showed that among the patients that presented as psoriatic spondylitis, the majority then developed peripheral disease of predominantly an oligo-articular pattern. A limitation of this study is its inability to differentiate whether this is a true representation of Psoriatic Spondylitis progression or if patients with PsA and inflammatory back pain without peripheral involvement escape referral to secondary care. Importantly, the delay in diagnosis of PsA with axial involvement is comparable with that of Ankylosing Spondylitis [2].

Patients with Psoriatic Spondylitis and Psoriatic Arthritis often develop clinical symptoms of disease and have normal blood tests. This along with lack of sacro-ilitis on imaging and the lack of genetic markers such as HLAB27 in this particular cohort may contribute to misdiagnosis. Diagnoses such as 'slipped disc', osteoarthritis or 'mechanical' reasons for the back pain may be given incorrectly. Additionally, 
it is known that the vast majority of patients with psoriatic spondylitis can be asymptomatic and preserve their spinal mobility for over ten years $[9,10]$. This delay in diagnosis that we have identified supports the literature and confirms this suspicion.

\section{Conclusion}

In our cohort, peripheral Psoriatic Arthritis is significantly more prevalent compared to Psoriatic Spondylitis.

The diagnosis is delayed in patients with Psoriatic Spondylitis compared to those with peripheral disease. However, this is comparable to the delay in diagnosing Ankylosing Spondylitis (mean delay 6 years).

Patients with peripheral Psoriatic Arthritis and Psoriatic Spondylitis have similar epidemiological characteristics. However; HLA B27 phenotype, Uveitis and high BASDAI score are more commonly associated with Psoriatic Spondylitis and earlier onset of disease.

More than $50 \%$ of patients in the Psoriatic Spondylitis group subsequently developed peripheral Arthritis. Of this group, the most common phenotype was that of oligo-articular disease. This raises the suitability of the exclusive classification of different Psoriatic Arthritic subtypes. These initial findings suggest that a disease spectrum may be more appropriate for Psoriatic Arthritis. This study suggests the need for further prospective observational studies with larger cohort, comparing peripheral and axial subtypes of Psoriatic Arthritis and reviewing disease progression over time, to elucidate this observation.

\section{References}

1. Gladman DD, Antoni C, Mease P et al. Psoriatic Arthritis: epidemiology, clinical features, course, and outcome. Ann. Rheum. Dis. 64, 14-7 (2005).

2. Nash P, Lubrano E, Cauli A et al. Updated Guidelines for the Management of Axial Disease in Psoriatic Arthritis. J. Rheumatol. 41, 2286-9 (2014).

3. Moll JMH, Wright V. Psoriatic arthritis. Semin. Arthritis. Rheum. 3, 55-78 (1973).

4. Taylor W, Gladman D, Helliwell P et al. CASPAR Study Group. Classification criteria for psoriatic arthritisdevelopment of new criteria from a large international study. Arthritis. Rheum. 54, 2665-73 (2006).

5. Roussou E, Chopra S, Ngandu DL. Phenotypic and clinical differences between Caucasian and South Asian patients with psoriatic arthritis living in North East London. Clin. Rheumatol. 32, 591-9 (2013).

6. Queiro R, Sarasqueta C, Belzunegui J et al. Psoriatic spondyloarthropathy comparative study between HLA-B27 positive and HLA-B27 negative disease. Semin. Arthritis. Rheum. 31, 413-8 (2002).

7. Rudwaleit $\mathrm{M}$, Heijde $\mathrm{D}$ van der, Landewé $\mathrm{R}$ et al. The development of Assessment of SpondyloArthritis international Society classification criteria for axial spondyloarthritis (part II): validation and final selection. Ann. Rheum. 68, 777-83 (2009).

8. Hanly JG, Mitchell MJ, Barnes DC et al. Early recognition of sacroiliitis by magnetic resonance imaging and single photon emission computed tomography. $J$. Rheumatol. 21, 2088-95 (1994).

9. Veale D, Rogers S, Fitzgerald O. Classification of clinical subsets in psoriatic arthritis. Br. J. Rheumatol. 33, 133138 (1994).

10. Chandran V, Barrett J, Schentag CT et al. Axial psoriatic arthritis: update on a longterm prospective study. J. Rheumatol. 36, 2744-2750 (2009). 